\title{
Remembering another aspect of forgetting
}

\section{Aaron M. Jasnow*, Patrick K. Cullen and David C. Riccio}

Department of Psychology, Kent State University, Kent, OH, USA

Edited by:

Oliver Hardt, McGill University,

Canada

Reviewed by:

Sven-Erik Fernaeus, Karolinska Institutet, Sweden

Einar Ö. Einarsson, INSERM

U862 - Neurocentre Magendie,

France

*Correspondence

Aaron M. Jasnow, Department of Psychology, Kent State University,

230 Kent Hall, Kent, OH 44242, USA

e-mail: ajasnow@kent.edu

\begin{abstract}
Although forgetting is most often thought of in terms of declines in performance (response loss or impairment), another class of memory phenomena, the forgetting of stimulus attributes, has begun to attract experimental attention. In non-human animals, the loss of memory for stimulus features is reflected in the flattening of stimulus generalization gradients as well as in the attenuation of the disrupting effect of a shift in context at testing. In both cases, a delay between the learning episode and testing results in increased responding in the presence of previously ineffective stimuli. Thus, previously discriminable cues become more functionally interchangeable. The implications of the forgetting of attributes for some theoretical issues of memory loss and for methodological strategies have been noted earlier. However, relatively little is known about the neurobiological mechanisms underlying stimulus attribute forgetting, and why some memories are maintained while others are not. In this paper we review the evidence for the forgetting of stimulus attributes, discuss recent findings identifying neurobiological underpinnings of forgetting and generalization of fear responses, and discuss relevant clinical implications of fear generalization.
\end{abstract}

Keywords: memory, fear, stimulus attributes, amygdala, hippocampus, context, generalization

\section{OVERVIEW}

As is generally known, conditioning to one stimulus (conditioned stimulus, CS) usually results in a tendency to respond to other similar stimuli as well, a phenomenon known as stimulus generalization. An important corollary to this principle is that the strength of responding is a direct function of the degree of similarity of test stimuli to the target CS. Thus, stimulus generalization is characterized by a gradient of responding, sometimes referred to as an "inverted V-shaped" function from its graphic representation. A steep generalization gradient would reflect sharply reduced levels of responding as the test (generalized) stimuli increasingly differ from the training cue. Conversely, responding to many generalized stimuli is reflected in a flatter gradient. An important but less well recognized aspect of generalization is that it tends to increase or flatten over time, such that previously ineffective generalized cues come to elicit conditioned responses. It seems clear that this increase in the range of effective cues is relevant to major clinical issues, including the spread of phobias and the impairments associated with PTSD.

\section{BACKGROUND}

Both Pavlov (1927) and Watson and Rayner (1920) had clearly identified stimulus generalization and recognized its evolutionary and adaptive importance. But that generalization is a temporally dynamic process appears not to have been recognized until a seminal study by Perkins and Weyant (1958), who found that generalization changed over a retention interval. In that experiment, performance of rats in an appetitively rewarded runway task was reduced (generalization decrement) when the color (black or white) of the runway was changed shortly after learning. In contrast, the same stimulus change after 1 week was much less disruptive - rats ran nearly as fast in the novel as in the training condition. Thus, subjects did not forget the motor response after the long interval but tended to respond similarly in both stimulus situations. As Perkins and Weyant presciently noted, “. . . forgetting the color of the runway occurs more rapidly than does the general tendency to run on elevated runways" (p. 599). Some years later Gisquet-Verrier and Alexinsky (1986), using runways painted flat white or white with black stripes, replicated and extended this finding by examining performance at several interpolated intervals. They found a systematic lessening of the disruption as a function of the retention intervals across 7 days. These changes in generalization are not attributable to a sensory or perceptual inability to detect the differences in the stimulus situations, as the impairment at the short retention intervals clearly indicates that the stimulus change could be recognized.

Shortly after the Perkins and Weyant (1958) report, several studies from D. R. Thomas' lab employed traditional operant conditioning procedures to investigate the effects of the training-totesting interval on stimulus generalization gradients. Consistent with the earlier work, Thomas found that the generalization gradient to a color dimension in pigeons became flatter as testing was delayed, although in these studies the major change was seen between an immediate and a $24 \mathrm{~h}$ test. Importantly, the flattening was not an artifact of a floor effect in performance, as subjects responded slightly more (not less) after the delay interval (e.g., Thomas and Lopez, 1962; Thomas and Burr, 1969).

All of these studies had involved only single stimulus training. Thus, one question is whether a similar effect would be obtained following discrimination training. Thomas et al. (1985) subsequently trained pigeons on a wavelength discriminations and found again that the generalization gradient flattened over time. 
It was also found that the change in the gradient took place more rapidly when the discriminative stimuli were close together, presumably because the more difficult discrimination also made it harder to remember the specific attributes of the stimuli during the test.

The generality of this type of forgetting is reflected in the evidence that it is obtained in a variety of paradigms in addition to those described above. For example, rats were found to avoid a greater range of sweet solutions after 7 and 10 days than after 2 days (Richardson et al., 1984). An indirect demonstration of forgetting of attributes comes from a study using a variation of the Kamin blocking effect. Substituting a generalized stimulus for the training CS shortly after conditioning did not block learning to the added element, but substituting the same stimulus did produce blocking after 3 weeks (Thomas and Riccio, 1979). Thus, the generalized stimulus came to function like the original CS.

The forgetting of attributes has also been obtained with stimuli prior to any training. In a latent inhibition paradigm involving conditioned taste aversion, pre-exposure to the target flavor did not affect conditioning to a generalized stimulus when the conditioning occurred 1 day later. However, latent inhibition to both the target CS and the generalized cue was seen after 8 days (Metzger and Riccio, 2009).

Increased responding to generalized cues can be seen as a form of "false positives." Thus, Bahrick et al. (1967) presented pictorial target stimuli (e.g., a teacup with a design) to college students. Subsequently, they were presented with an array of items ranging in similarity to the target and asked to choose the original stimulus. With increasing retention intervals the subjects tended to incorrectly pick generalized cues rather than the training stimulus.

\section{GENERALIZATION OF CONTEXTUAL STIMULI}

That features of stimuli associated with a learning episode are forgotten more rapidly than the conditioned response itself is not limited to the conditioned or discriminative stimuli, but can include contextual cues. As Spear (1973) noted some years ago, it is useful to conceptualize memory not simply as a unitary trace, but rather as a representation consisting of a number of different attributes that include contexts as well as the more specific elements of conditioning. Historically, contextual stimuli referred to background stimuli present at the time of learning but not directly relevant to the task. Associations to these background cues can be seen as a process similar to what has been called "incidental learning" in human research literature. In incidental learning, human subjects are instructed to learn a particular set of materials (e.g., paired-associates) but later are also tested for information that they may have acquired about stimuli present at the time of learning the target task. In the non-human animal studies discussed below, the subjects are queried about contextual associations by changing the context in which testing occurs.

It should be noted that in recent years the term "context" has been broadened to include learning paradigms in which no discrete or punctuate stimulus such as a tone is introduced. In this case, although the only predictive stimulus is the context itself, the distinguishing feature is that typically the contextual cues are static and present both before and after the presentation of any unconditioned stimulus. It should also be recognized that "context" is a very general term that consists of a number of different stimuli such as spatial array, odors, ambient noise, and illumination, etc.

In a Pavlovian fear conditioning task where a light (CS) predicted shock, rats were later tested for escape responding to that same signal in either the training chamber or an apparently "identical" but different chamber. Performance was impaired in the different apparatus on an immediate test, indicating a context-specific memory. That is, the change in context presumably removed cues that were needed for retrieval of the fear memory, resulting in memory for the predictive value of the CS that was limited to the training chamber (context). However, responding was comparable in both chambers after a 1 day retention interval (McAllister and McAllister, 1963). Importantly, this outcome revealed that the forgetting of stimulus attributes is also applicable to contextual stimuli and indicated that the novel contextual test cues had come to function as retrieval cues.

Other evidence for the forgetting of contextual attributes comes from a study on passive avoidance in rats, a task in which the avoidance response is particularly well retained over time. Zhou and Riccio (1996) examined some aspects of context and found that changes in either the apparatus, or the room, or both, produced a profound impairment in avoidance with a 1-day retention test interval, i.e., the context shift effect. However, after 2 weeks the groups with only one component changed performed as well as the group tested in the same (training) context. The impairment persisted in the condition where both components were changed, presumably because with extremely different stimuli either the forgetting does not occur or would take longer than the 2-week delay used in that experiment. More recently, Biedenkapp and Rudy (2007) found a context shift effect 1 day but not 7 or 15 days after fear conditioning. Furthermore, in a second experiment in which they increased the number of context pre-exposures, they were able to increase the length of the retention interval where the disruptive effect of context change was obtained. This finding supports the view that memory for contextual representations follows other learning principles - stronger representations are more persistent over time.

Although many of the studies of the forgetting of attributes described above used rats as subjects, the effect has been obtained repeatedly in mice as well (Wiltgen and Silva, 2007; Wiltgen et al., 2010; Ruediger et al., 2011). For example, Wiltgen and Silva (2007) showed that when mice were tested 1 day after fear conditioning to a context, performance was impaired in a different context. However, this shift effect diminished systematically as a function of increases in the delay of test interval over 14, 28, or 36 days. Similarly, Ruediger et al. (2011) found little generalization of fear between the training and novel contexts after 1 or 7 days, but substantial freezing to the new context with retention intervals of 15 and 40 days.

In another experiment to rule out the possible effects of fear incubation, Wiltgen and Silva (2007) exposed mice to the context either 1 or 36 days prior to conditioning. All subjects were then tested 1 day after training, thus holding constant the fear conditioning to retention interval. Again, fear generalized to the novel context after the long but not the short interval.

The forgetting of contextual features is found for positively reinforced learning as well as fear conditioning. For example, using 
a latent inhibition paradigm in Pavlovian appetitive conditioning, Rosas and Bouton (1997) examined the effects of context changes on conditioning. A change in context eliminated the latent inhibition effect when training began at a short interval after CS pre-exposure but not after a long (28 day) interval. Winocur et al. (2007) used food preference conditioning to evaluate context effects. Rats preferences were impaired by a change in context at 1 day but not at 8 days.

A study from Rovee-Collier's lab with human infants several months of age used crib liners as the context in which the infants learned to activate a mobile (reinforcement) by kicking their leg. A change in the crib liner 1 day after conditioning was disruptive, indicating that the context was perceived and encoded. However, the same change became increasingly less disruptive as the retention interval increased (Borovsky and Rovee-Collier, 1990). A developmental study with rats compared the forgetting of contextual attributes in pre-weanling (18 day old) pups and adults. The context shift effect was obtained in both age groups at a $1 \mathrm{~h}$ interval. After $48 \mathrm{~h}$, however, only the adult rats distinguished between the two contexts, as reflected in impaired performance in the shifted condition (Anderson and Riccio, 2005).

Although most of the research on contextual stimuli has involved external background cues, a study by MacArdy asked whether forgetting of attributes might also occur for internal cues. Rats received fear conditioning in the presence of one drug as the internal context and were tested later after administration of the same or a different, but related, drug. At a 1-day interval the change in drug state resulted in impaired responding. However, the impairment was not found when the different drug state was present after a 1-week retention interval (MacArdy and Riccio, 1991).

As these studies indicate, the importance of the forgetting of attributes is that with the passage of time a greater range of stimuli can come to elicit or control conditioned responding (e.g., Riccio et al., 1994). Both the conceptual and methodological implications of this form of forgetting have been reviewed earlier so we will only briefly mention them here.

With respect to forgetting of responses, changes in context at testing from that at acquisition, or "altered stimulating conditions," have long been considered one of two major sources of retention loss (e.g., McGeoch, 1942; Spear, 1973; Spear and Riccio, 1994). While it seems reasonable that over time subtle changes in contexts from the learning episode are likely to occur, the so called "contextual cues paradox" is that fairly large and explicit changes in context have less disruptive effect on performance after a long interval (Riccio et al., 1984). Thus, it would seem difficult to explain impaired performance after long intervals on the basis of a naturally occurring change in context (cf., Bouton et al., 1999; Riccio et al., 1999). But, it should be noted that the increased functional similarity of contextual cues would afford an opportunity for increases in retroactive interference from learning in other situations.

An important limitation on the effectiveness of extinction in eliminating responses is the renewal effect (e.g., Bouton and Bolles, 1979). In renewal, a response eliminated through extinction in a context different from that at learning recovers or "renews" when subjects are returned to the training context. The clinical implications of renewal, especially with respect to the extinction of fear, have been aptly noted by Bouton (2002). The forgetting of contextual attributes makes the interesting prediction (now being tested) that the passage of time should attenuate renewal.

Methodologically, to determine the role of associations in various phenomena, one strategy involves altering the context or other stimuli. Thus, it is important to recognize that the outcome obtained may depend on the time (usually arbitrarily chosen) until testing. For example, Feinberg and Riccio (1990) replicated the valuable work by Siegel $(1975,1976)$ showing that morphine tolerance depended on associative processes: Tolerance was eliminated when testing at 1 day occurred in a context different from where the drug had been repeatedly administered. But if the test session was delayed by a week, the change in context had no effect. Had only the delayed test been given the outcome could erroneously lead to the conclusion that tolerance was based on pharmacological rather than associative mechanisms (Riccio et al., 1992).

One other implication of the forgetting of attributes to be considered pertains to "false memories." False memories are usually considered to be memories for an event (or word) that did not occur (for review see Roediger and McDermott, 1996). However, increased responding over time to stimuli that were not present at the time of an event might also be seen as a form of false memory. For example, after a long retention interval many cues that are quite different from the training stimulus result in fear elicited freezing in rodents. Essentially, then, subjects are behaving as if the generalized cues were, in fact, part of the original episode.

\section{REINSTATEMENT OF MEMORY FOR STIMULUS ATTRIBUTES}

As is the case with the more traditional issue of the forgetting of responses, an important question is whether impairment of memory for attributes can be reversed or alleviated. An early study in the operant tradition showed that reminders in the form of brief exposure to an element of training (but not involving retraining) could re-sharpen the stimulus generalization gradient in pigeons (Moye and Thomas, 1982).

As part of their important investigation of whether contextual change is the basis for long-term deficits in response performance, Gisquet-Verrier and Alexinsky (1986) examined the effects of a brief exposure to the experimental context after a 2 week retention interval. In the absence of the contextual reminder, performance of a running response for food was comparable in both the training and novel test contexts. The reminder, however, reinstated the context change effect, such that performance in the new context was now impaired, much as is the case at a short retention interval. In a related study using passive avoidance as the indicator of context effects, Zhou and Riccio (1994) obtained a similar result: After a long delay interval, exposure to the training context (without further shocks) before testing reinstated the disruptive effect of a context change. In addition, that experiment found that the return of attribute memory was relatively transient such that the reminder had no effect if testing was delayed by several hours. Using mice, Wiltgen and Silva (2007) replicated the finding that a brief reminder in the form of exposure to the training context after a retention interval was sufficient to reinstate the contextual discrimination. 
In an extensive study of the role of the growth of feed forward inhibitory connections in regulating memory "precision" in mice, Ruediger et al. (2011) found that re-exposure to the training context after 15 days greatly reduced the generalization to context. A control condition showed that exposure to a neutral or unrelated context had no effect on broadened contextual generalization. Unlike the Zhou and Riccio (1994) study, the reinstatement of attribute memory persisted for some 8-12 days (Ruediger et al., 2011), a disparity that might be accounted for by any number of the many methodological differences between the experiments.

In their study of contextual change and latent inhibition (described above), Rosas and Bouton (1997) showed that altering the context after a long interval did not disrupt the effect of pre-exposure to the CS. However, a brief re-exposure to the context at the end of the interval and shortly prior to training reinstated the context shift effect, i.e., latent inhibition was attenuated in the presence of the altered context, very much like the effect at the short interval.

These outcomes raise intriguing questions about how contextual memory is stored, if it can be recovered in its original form through reminders that do not involve retraining. Furthermore, although reinstating the precision of memory has received even less attention in the research literature than the forgetting of attributes, the phenomenon is potentially important in understanding the underlying biological mechanisms of this form of memory impairment (see below). Furthermore, procedures to reduce generalization could well have useful implications for treating anxiety disorders, since the "spread" of fear to stimuli not directly associated with a traumatic incident is one of the debilitating aspects of anxiety related pathology such as PTSD.

\section{BEHAVIORAL AND NEUROBIOLOGICAL MECHANISMS IN FORGETTING ATTRIBUTES}

Despite the importance of forgetting of attributes, and in contrast with decades of research on forgetting of learned responses, surprisingly little research has examined the behavioral and physiological processes that underlie this type of memory loss. A study by Smith (1979) indirectly suggests that interference from other stimuli is likely to play a role. Having shown that the impairment resulting from a context change at a short interval was alleviated by instructing college students to recall the context in which material had been learned, he found that this reminder manipulation was ineffective if the students were exposed to several other contexts prior to testing.

Wixted (2004), in an extensive review of the literature, has suggested that, contrary to the generally accepted view, retroactive interference has a time dependent characteristic similar to retrograde amnesia. Moreover, he proposes that interference effects can be produced by learning unrelated material, not simply from directly competing responses as in the typical design. Given the dearth of behavioral data on forgetting of attributes, Wixted's views have interesting implications. Thus, following conditioning in one context, exposure to multiple contexts without further training should lead to faster forgetting of the original context, as reflected in the attenuation of the context change effect. Intriguingly, the interfering effects of the multiple contextual exposures should be more pronounced when the manipulation is introduced shortly after learning than after a long interval.

\section{CONTEXT GENERALIZATION}

Only recently have some investigators begun to explore the biological substrates for the changes in generalization as a function of increases in the retention interval. Several recent studies provide important contributions to understanding the neurobiological mechanisms underlying generalization of memory. All of these studies replicate the basic finding that when rodents receive footshocks in one context and are tested in a different context $24 \mathrm{~h}$ later they observe a decrease in freezing (the context shift effect). However, when rodents are tested at long delays after training, the context shift effect is eliminated; rodents freeze equivalently to the training and novel contexts (Riccio et al., 1984; Zhou and Riccio, 1996). Several hypotheses of what happens to information over time have implications for how the mechanisms underlying generalization of contextual cues are interpreted. Systems consolidation suggests that during memory consolidation, hippocampal activation is involved in retrieving newly acquired information about the context, but over time, storage of information about specific aspects of the context becomes distributed to neocortical sites (Squire, 1992; Alvarez et al., 1995; Frankland et al., 2001, 2004). In this view, the hippocampus is necessary for retaining contextdependent information at short intervals, but not at long intervals. This has recently been illustrated by data suggesting that the anterior cingulate cortex (ACC) is necessary for the recall of remote, but not recent memories. Using activity-dependent gene expression analysis, Frankland et al. (2004) demonstrated that retrieval of recent information involves activation of the hippocampus, whereas retrieval (or retrieval attempts) at longer retention intervals engages a distributed neocortical network, including the ACC, infralimbic, and prelimbic cortices. Pharmacological inactivation of the ACC disrupted remote contextual fear memory, but had no effect on recent fear, suggesting that neocortical circuits are necessary for remote memory retrieval, of which the ACC is an essential structure. It should be noted, however, that these experiments did not examine the specificity of the memory performance at recent and remote time points. While they are informative about the limited role of the hippocampus for remote memories, they do not explain the generalization of contextual memories as they age.

In an attempt to explain this phenomenon, and as inferred from changes in performance, Winocur et al. (2007) suggested that memory traces may be transformed during storage in the cortex to become less specific over time and also context-independent (Winocur et al., 2007). In this study they lesioned the hippocampus to examine its role in the generalization to contextual cues. Using this approach they found that the context shift at the short interval was eliminated by hippocampal lesions (see also Penick and Solomon, 1991). Equivalent performance between the same and shifted context groups at a long delay in hippocampal lesioned rats was interpreted as being consistent with the transformational model. In other words, information about the training context becomes modified during storage into a more 
schematic (generalized or gist) representation in the cortex over time. These interpretations, however, were based on data obtained from rats with pre-training lesions, making this conclusion difficult because of the impaired performance displayed by rats tested in the same context as the training context. An additional study (Winocur et al., 2009), using post-training lesions demonstrated that the hippocampus was not involved in generalized contextual fear memories. Next, they found that hippocampal lesions eliminated freezing to the training and to a general context, when rats were given a reminder of the training context. However, hippocampal lesions did not eliminate freezing to either the training or general context when rats were given a reminder in a general context. This was interpreted as evidence for two memory traces; a memory trace that was specific for the training context involving the hippocampus and a transformed memory trace mediated by neocortical areas.

Recently, Wiltgen et al. (2010) demonstrated that the hippocampus was selectively involved in detailed contextual memories. These data and data from a more recent study by Ruediger et al. (2011), suggest that the hippocampus is necessary for longterm memory for as long as that memory is context-dependent. These findings are inconsistent with the systems consolidation view of long-term memory, and instead support the transformation view of memory consolidation. The study by Ruediger et al. (2011) is particularly interesting because it illustrates some of the molecular and cellular mechanisms that may underlie the role of the hippocampus in memory precision. They demonstrated that context fear learning induces selective increases of filopodial contacts onto parvalbumin-expressing fast spiking interneurons in the CA3 region, thereby enhancing feedforward inhibition. As the context memory trace aged, the number of filopodial contacts decreased, the mice began to freeze to a novel context (i.e., loss of the context shift effect). Additionally, mice lacking the Rab3a gene ( $R a b 3 a^{-/-}$), a GTP-binding synaptic vesicle protein involved in calcium-dependent exocytosis, fear conditioned normally, but generalized their fear response at 1 day. These mice also lacked long-term potentiation (LTP) at CA3 synapses and any feedforward inhibitory growth. Theses data suggest that synaptic plasticity is necessary for the growth of feedforward inhibitory filopodia and maintenance of a precise memory. Additionally, Add2-1mice, which exhibit early LTP, but cannot stabilize synapses, were able to learn context fear normally, but generalized after 1 day. Although these mice exhibit early LTP, they also did not exhibit feedforward inhibitory growth. Taken together, these data suggest that feedforward inhibitory growth in the CA3 region of the hippocampus is necessary for maintaining precise memories over time, but not necessary for establishing the memory itself.

It is clear from a number of studies that the hippocampus mediates context-specific memory and over time the memory trace becomes consolidated in the cortex as a more schematic or gistlike representation (but see Biedenkapp and Rudy, 2007). It is not clear, however, what structures are involved in generalized memory. Based on the studies using immediate early gene expression and metabolic activity during remote memory retrieval (a time when a schematic representation would dominate), as well as temporary region specific inactivation (Bontempi et al., 1999;
Frankland et al., 2004; Maviel et al., 2004; Wiltgen et al., 2010), the ACC might be expected to be important in regulating context generalization. In addition to questions on regional control of generalized memories, the specific mechanisms that regulate transformation of a context-specific memory trace within the hippocampus to a context-general memory trace in cortical structures are entirely unknown. Thus, despite a number of important studies demonstrating transformation of specific contextual information in the hippocampus to schematic or gist-like information in the neocortex, the precise neocortical regions and underlying mechanisms of context generalization are still unclear. Two recent studies discussed above shed light on this, suggesting that the hippocampus is involved in memory for as long as that memory is context-dependent (Wiltgen et al., 2010; Ruediger et al., 2011).

\section{CUE GENERALIZATION}

It has generally been recognized that cue-dependent and context fear memory are regulated by overlapping, yet different neurobiological mechanisms (Phillips and LeDoux, 1992; Frankland et al., 1998; Maren and Holt, 2004; Fanselow, 2010). Specifically, the amygdala is essential in the acquisition, consolidation, and extinction of cue-dependent and contextual fear, whereas the hippocampus is generally thought to be essential in the regulation of contextual fear (Kim et al., 1993; Fanselow and Kim, 1994; Helmstetter and Bellgowan, 1994; Maren, 1999; Davis and Aggleton, 2000; Schafe et al., 2001; Walker et al., 2002; Phelps et al., 2004; Myers, 2006). Several recent studies have examined the mechanisms underlying cued fear generalization using cued discrimination training. These studies find that mice lacking the $65 \mathrm{kD}$ form of glutamic-acid decarboxylase (GAD65 $5^{-1-}$ mice) which is the biosynthetic enzyme for $\gamma$-aminobutyric acid (GABA), display reduced freezing compared to their wild type littermates, suggesting reduced fear memory. Interestingly, GAD65 ${ }^{-1-}$ mice were unable to distinguish the CS- from the CS+ during testing at $24 \mathrm{~h}$ or 14 days, suggesting that GABA synthesis is not only critical for appropriate fear memory, but also for stimulus specific fear memory (Bergado-Acosta et al., 2008). In addition to $\mathrm{GABA}$ synthesis, presynaptic $\mathrm{GABA}_{\mathrm{B}}$ receptors also appear to play an important role in fear generalization. Pharmacological blockade of $\mathrm{GABA}_{\mathrm{B}}$ receptors in the lateral amygdala allows the induction of homosynaptic LTP at cortical afferents when using stimulation protocols that normally do not elicit LTP (Shaban et al., 2006). Two $\mathrm{GABA}_{\mathrm{B}(1)}$ subunit isoforms are expressed in the brain, $\mathrm{GABAB}_{1(\mathrm{a})}$, which is selectively associated with presynaptic terminals, and $\mathrm{GABAB}_{1(\mathrm{~b})}$ which is associated with postsynaptic membranes (Vigot et al., 2006). In the lateral amygdala, $\mathrm{GABAB}_{1(\mathrm{a})}$ receptors seem to be specifically responsible for presynaptic LTP at cortico-amygdala afferents, whereas, $\mathrm{GABAB}_{1(\mathrm{~b})}$ receptors are responsible for LTP at thalamic afferents, suggesting pathway-specific regulation of LTP in the lateral amygdala (Shaban et al., 2006). Moreover, $\mathrm{GABAB}_{1(\mathrm{a})}{ }^{-1-}$ mice display stimulus-intensity dependent generalization of fear to CS- cues when tested after discrimination training. These data suggested that presynaptic inhibition in the cortico-amygdala pathway is important to preserve stimulus discrimination during discrimination training. Additionally, the lack of presynaptic inhibition 
may shift the threshold for generalization of fear to lower US intensities.

In addition to the lateral amygdala and basolateral amygdala, the central nucleus of the amygdala (CeA) has been identified as an essential structure undergoing plasticity in support of fear learning (Jasnow and Huhman, 2001; Samson and Pare, 2005; Wilensky et al., 2006). The CeA is predominantly composed of GABA-ergic inhibitory neurons, of which, the medial subdivision of the $\mathrm{CeA}(\mathrm{Cem})$ is under tonic inhibition by the lateral/capsular CeA (Cel; Cassell et al., 1986; Sun and Cassell, 1993; Ciocchi et al., 2010; Haubensak et al., 2010). Using a discrimination task and in vivo electrophysiological recordings, Ciocchi et al. (2010) showed that a decrease in tonic activity of Cem neurons was associated with generalization to the CS-. The opposite was true for the Cel; an increase in tonic activity was associated with generalization. In addition, changes in tonic activity of the Cel were higher in generalizing mice. These data suggest that specificity of fear responses may be partially regulated by changes in activity within the inhibitory neural circuitry of the CeA. Changes in activity between these subnuclei may regulate the signal-to-noise ratio of fear stimuli, and thus play a role in stimulus specific behavioral output (Ciocchi et al., 2010). Generalization to fearful cues may also recruit activity of additional brain regions, including the bed nucleus of the stria terminalis (BNST). BNST lesioned rats froze less to the CS- and were able to discriminate between a $\mathrm{CS}+$ and CS - whereas, sham lesioned rats display a continuum of responses, ranging from high to low generalization (Duvarci et al., 2009). Based on the limited available data, it appears that the inhibitory network within the amygdala plays an essential role in regulating stimulus specific behavioral responses, and ultimately limits generalization. In addition, recruitment of the BNST, which is involved in regulating anxiety, may contribute to fear generalization. This is also true for generalization of behavioral responses following social defeat. In social defeat models, animals are defeated in one context by one conspecific individual and then tested in another context with a novel stimulus conspecific (Huhman et al., 2003; Jasnow et al., 2004). Modulation of BNST activity leads to reduced behavioral effects of defeat when animals are tested in an alternative context and with a novel stimulus animal (Jasnow et al., 2004). It must be noted that many of these studies discussed above used discrimination training, which does not address the question of generalization to novel cues. However, it is likely that generalization to novel cues involves similar neurobiological mechanisms as described in the studies above.

Contextual fear generalization likely involves transformation of specific contextual information in the hippocampus to schematic or gist-like information in the neocortex. Do cued fear memories undergo a similar transformation as the memory trace ages from a specific to general representation as it does for contextual memories? If so, are the same neocortical brain regions (ACC, infralimbic, and prelimbic) involved in the generalized memory? The amygdala is a cortical-like structure with similar intrinsic circuitry but lacks laminar organization (McDonald, 1989, 1996; McDonald and Pearson, 1989; McDonald and Mascagni, 2001, 2002; Mascagni and McDonald, 2003; Jasnow et al., 2009).
Several lines of evidence suggest that the lateral and/or basolateral complex of the amygdala may be the locus of fear memory storage (Schafe et al., 2005; Kwon and Choi, 2009; Poulos et al., 2009). Alternatively, the amygdala may modulate memories that are stored in the cortex (McGaugh, 2004; Chavez et al., 2009). Another possibility is that cued fear memories generalize as loss of feedforward inhibition onto parvalbumin-expressing interneurons occurs, similar to what has been observed in the hippocampus (Ruediger et al., 2011). Thus, it is possible that cued fear memories undergo a transformation from cue-specific to cuegeneral representation within the same region and may occur at a faster rate than context memories. Alternatively, generalization of cued fear may be due to changes in afferent or efferent structures with direct connections with the amygdala, such as the auditory thalamus or auditory cortex (Weinberger, 2007). In the case of the auditory thalamus, enhanced fear generalization to alternative tones that were not previously paired with shock has been observed following increased cAMP-response element binding protein (CREB) over-expression in the medial geniculate nucleus (Han et al., 2008). This would enable relatively quick generalization of fear responses to alternate cues, as broadening of auditory thalamus tuning may occur during, or shortly after conditioning.

\section{CONCLUDING REMARKS}

The forgetting of stimulus attributes is not a new phenomenon, nor for that matter is the increase in performance associated with this type of forgetting for some behavioral tasks. What is relatively new is the increased attention paid to the neurobiological mechanisms underlying the forgetting of stimulus attributes, particularly in reference to fear generalization. Using this learning model it has become apparent that the hippocampus plays an important role in memory traces that involve specific contextual information, whereas, neocortical brain regions play a particularly important role in schematic or gist-like memory traces. This transformation seems to happen as memories age. However, specific examination of which neocortical structures are necessary for schematic or gist-like fear memory has not yet been published. Specific structural changes within the hippocampus leading to increased feedforward inhibition may underlie the mechanism by which this structure maintains a precise memory trace. It is currently unclear whether generalization of fear responses to specific cues involves similar mechanisms to generalization of contextual fear. However, GABA-ergic mechanisms within the amygdala appear to play an important role in maintaining a precise cued fear memory, somewhat similar to the role of feedforward inhibition in the hippocampus. Given that generalized fear is a hallmark of several neuropsychiatric emotional disorders, including PTSD, understanding the neurobiological mechanisms of generalized fear and its expansion with the passage of time will be critical to developing effective treatments for these disorders. Convergent data from both non-human animal models of fear and concurrent studies in humans will allow a more robust understanding of the environmental, genetic, and neurobiological mechanisms underlying generalized fear and it's contribution to a number of neuropsychiatric disorders of emotion. 


\section{REFERENCES}

Alvarez, P., Zola-Morgan, S., and Squire, L. R. (1995). Damage limited to the hippocampal region produces long-lasting memory impairment in monkeys. J. Neurosci. 15, 3796-3807.

Anderson, M. J., and Riccio, D. C. (2005). Ontogenetic forgetting of stimulus attributes. Learn. Behav. 33 , 444-453.

Bahrick, H. P., Clark, S., and Bahrick, P. (1967). Generalization gradients as indicants of learning and retention of a recognition task. J. Exp. Psychol. 75, 464-471.

Bergado-Acosta, J. R., Sangha, S., Narayanan, R. T., Obata, K., Pape, H. C., and Stork, O. (2008). Critical role of the $65-\mathrm{kDa}$ isoform of glutamic acid decarboxylase in consolidation and generalization of Pavlovian fear memory. Learn. Mem. 15, 163-171.

Biedenkapp, J. C., and Rudy, J. W. (2007). Context preexposure prevents forgetting of a contextual fear memory: implication for regional changes in brain activation patterns associated with recent and remote memory tests. Learn. Mem. 14, 200-203.

Bontempi, B., Laurent-Demir, C., Destrade, C., and Jaffard, R. (1999). Time-dependent reorganization of brain circuitry underlying longterm memory storage. Nature 400 , 671-675.

Borovsky, D., and Rovee-Collier, C. (1990). Contextual constraints on memory retrieval at six months. Child Dev. 61, 1569-1583.

Bouton, M. E. (2002). Context, ambiguity, and unlearning: sources of relapse after behavioral extinction. Biol. Psychiatry 52, 976-986.

Bouton, M. E., and Bolles, R. C. (1979). Contextual control of the extinction of conditioned fear. Science 10, 445-466.

Bouton, M. E., Nelson, J. B., and Rosas, J. M. (1999). Stimulus generalization, context change, and forgetting. Psychol. Bull. 125, 171-186.

Cassell, M. D., Gray, T. S., and Kiss, J. Z. (1986). Neuronal architecture in the rat central nucleus of the amygdala: a cytological, hodological, and immunocytochemical study. $J$. Comp. Neurol. 246, 478-499.

Chavez, C. M., McGaugh, J. L., and Weinberger, N. M. (2009). The basolateral amygdala modulates specific sensory memory representations in the cerebral cortex. Neurobiol. Learn. Mem. 91, 382-392.

Ciocchi, S., Herry, C., Grenier, F., Wolff, S., Letzkus, J., Vlachos, I., Ehrlich, I., Sprengel, R., Deisseroth, K., Stadler, M., Muller, C., and Luthi, A. (2010).
Encoding of conditioned fear in central amygdala inhibitory circuits. Nature 468, 277-282.

Davis, M., and Aggleton, J. (2000). The role of the amygdala in conditioned and unconditioned fear and anxiety. Ann. Rev. Neurosci. 353-375.

Duvarci, S., Bauer, E., and Pare, D. (2009). The bed nucleus of the stria terminalis mediates inter-individual variations in anxiety and fear. $J$. Neurosci. 29, 10357-10361.

Fanselow, M. S. (2010). From contextual fear to a dynamic view of memory systems. Trends Cogn. Sci. (Regul. Ed.) $14,7-15$.

Fanselow, M. S., and Kim, J. J. (1994). Acquisition of contextual Pavlovian fear conditioning is blocked by application of an NMDA receptor antagonist D,L2-amino-5-phosphonovaleric acid to the basolateral amygdala. Behav. Neurosci. 108, 210-212.

Feinberg, G., and Riccio, D. C. (1990). Changes in memory for stimulus attributes: implications for tests of morphine tolerance. Psychol. Sci. 1, 265-267.

Frankland, P., O'Brien, C., Ohno, M., Kirkwood, A., and Silva, A. (2001). $\alpha$-CaMKII-dependent plasticity in the cortex is required for permanent memory. Nature 411, 309-313.

Frankland, P. W., Bontempi, B., Talton, L. E., Kaczmarek, L., and Silva, A. J. (2004). The involvement of the anterior cingulate cortex in remote contextual fear memory. Science 304, 881-883.

Frankland, P. W., Cestari, V., Filipkowski, R. K., McDonald, R. J., and Silva, A. J. (1998). The dorsal hippocampus is essential for context discrimination but not for contextual conditioning. Behav. Neurosci. 112, 863-874.

Gisquet-Verrier, P., and Alexinsky, T. (1986). Does contextual change determine long-term forgetting? Animal Learn. Behav. 14, 349-358.

Han, J. H., Yiu, A. P., Cole, C. J., Hsiang, H. L., Neve, R. L., and Josselyn, S. A. (2008). Increasing CREB in the auditory thalamus enhances memory and generalization of auditory conditioned fear. Learn. Mem. 15, 443-453.

Haubensak, W., Kunwar, P. S., Cai, H., Ciocchi, S., Wall, N. R., Ponnusamy, R., Biag, J., Dong, H. W., Deisseroth, K., Callaway, E. M., Fanselow, M. S., Luthi, A., and Anderson, D. J. (2010). Genetic dissection of an amygdala microcircuit that gates conditioned fear. Nature 468 270-276.
Helmstetter, F. J., and Bellgowan, P. S. (1994). Effects of muscimol applied to the basolateral amygdala on acquisition and expression of contextual fear conditioning in rats. Behav. Neurosci. 108, 1005-1009.

Huhman, K., Solomon, M., Janicki, M., Harmon, A., Lin, S., and Jasnow, A. (2003). Conditioned defeat in male and female syrian hamsters. Horm. Behav. 44, 293-299.

Jasnow, A., and Huhman, K. (2001). Activation of GABA(A) receptors in the amygdala blocks the acquisition and expression of conditioned defeat in Syrian hamsters. Brain Res. 920 142-150.

Jasnow, A., Huhman, K., and Davis, M. (2004). Involvement of central amygdalar and bed nucleus of the stria terminalis corticotropinreleasing factor in behavioral responses to social defeat. Behav. Neurosci. 118, 1052-1061.

Jasnow, A. M., Ressler, K. J., Hammack, S. E., Chhatwal, J. P. and Rainnie, D. G. (2009). Distinct subtypes of cholecystokinin (CCK)-containing interneurons of the basolateral amygdala identified using a CCK promoter-specific lentivirus. J. Neurophysiol. 101, 1494-1506.

Kim, J. J., Rison, R. A., and Fanselow, M. S. (1993). Effects of amygdala, hippocampus, and periaqueductal gray lesions on short and long-term contextual fear. Behav. Neurosci. 107, 1093-1098.

Kwon, J. T., and Choi, J. S. (2009). Cornering the fear engram: longterm synaptic changes in the lateral nucleus of the amygdala after fear conditioning. J. Neurosci. 29, 9700-9703.

MacArdy, E. A., and Riccio, D. C. (1991) Increased generalization between drug-related interoceptive stimuli with delayed testing. Behav. Neural Biol. 56, 213-219.

Maren, S. (1999). Neurotoxic basolateral amygdala lesions impair learning and memory but not the performance of conditional fear in rats. J. Neurosci. 19, 8696-8703.

Maren, S., and Holt, W. (2004). Hippocampus and Pavlovian fear conditioning in rats: muscimol infusions into the ventral, but not dorsal, hippocampus impair the acquisition of conditional freezing to an auditory conditional stimulus. Behav. Neurosci. 118, 97-110.

Mascagni, F., and McDonald, A. (2003). Immunohistochemical characterization of cholecystokinin containing neurons in the rat basolateral amygdala. Brain Res. 976, 171-184.

Maviel, T., Durkin, T. P., Menzaghi, F., and Bontempi, B. (2004). Sites of neocortical reorganization critical for remote spatial memory. Science 305, 96-99.

McAllister, W. R., and McAllister, D. E. (1963). Increase over time in the stimulus generalization of acquired fear. J. Exp. Psychol. 65, 576.

McDonald, A. (1989). Coexistence of somatostatin with neuropeptide Y, but not with cholecystokinin or vasoactive intestinal peptide, in neurons of the rat amygdala. Brain Res. 500, 37-45.

McDonald, A. (1996). Glutamate and aspartate immunoreactive neurons of the rat basolateral amygdala: colocalization of excitatory amino acids and projections to the limbic circuit. J. Comp. Neurol. 365, 367-379.

McDonald, A., and Mascagni, F. (2001). Colocalization of calcium-binding proteins and GABA in neurons of the rat basolateral amygdala. Neuroscience 105, 681-693.

McDonald, A., and Mascagni, F. (2002). Immunohistochemical characterization of somatostatin containing interneurons in the rat basolateral amygdala. Brain Res. 943, 237-244.

McDonald, A., and Pearson, J. (1989). Coexistence of GABA and peptide immunoreactivity in non-pyramidal neurons of the basolateral amygdala. Neurosci. Lett. 100, 53-58.

McGaugh, J. L. (2004). The amygdala modulates the consolidation of memories of emotionally arousing experiences. Annu. Rev. Neurosci. 27, $1-28$.

McGeoch, J. A. (1942). The Psychology of Human Learning. New York: Longmans, Green.

Metzger, M. M., and Riccio, D. C. (2009). The forgetting of stimulus attributes in latent inhibition. Physiol. Behav. 96, 194-198.

Moye, T. B., and Thomas, D. R. (1982). Effects of memory reactivation treatments on postdiscrimination generalization performance in pigeons. Learn. Behav. 10, 159-166.

Myers, K. M. (2006). Different mechanisms of fear extinction dependent on length of time since fear acquisition. Learn. Mem. 13, 216-223.

Pavlov, I. (1927). Conditioned Reflexes: An Investigation of the Physiological Processes of the Cerebral Cortex. London: Oxford University Press.

Penick, S., and Solomon, P. R. (1991). Hippocampus, context, and conditioning. Behav. Neurosci. 105, 611-617. 
Perkins, C. C. Jr., and Weyant, R. G. (1958). The interval between training and test trials as a determiner of the slope of generalization gradients. J. Comp. Physiol. Psychol. 51, 596-600.

Phelps, E. A., Delgado, M. R., Nearing, K. I., and LeDoux, J. E. (2004). Extinction learning in humans. Neuron 43, 897-905.

Phillips, R. G., and LeDoux, J. E. (1992). Differential contribution of amygdala and hippocampus to cued and contextual fear conditioning. Behav. Neurosci. 106, 274-285.

Poulos, A. M., Li, V., Sterlace, S. S. Tokushige, F., Ponnusamy, R., and Fanselow, M. S. (2009). Persistence of fear memory across time requires the basolateral amygdala complex. Proc. Natl. Acad. Sci. U.S.A. 106, 11737-11741.

Riccio, D. C., Ackil, J., and BurchVernon, A. (1992). Forgetting of stimulus attributes: methodological implications for assessing associative phenomena. Psychol. Bull. 112, 433-445.

Riccio, D. C., Rabinowitz, V. C., and Axelrod, S. (1994). Memory: when less is more. Am. Psychol. 49, 917-926.

Riccio, D. C., Richardson, R., and Ebner, D. L. (1984). Memory retrieval deficits based upon altered contextual cues: a paradox. Psychol. Bull. 96, 152-165.

Riccio, D. C., Richardson, R., and Ebner, D. L. (1999). The contextual change paradox is still unresolved: comment on Bouton, Nelson, and Rosas (1999). Psychol. Bull. 125, 187-189.

Richardson, R., Williams, C., and Riccio, D. C. (1984). Stimulus generalization of conditioned taste aversion in rats. Behav. Neural Biol. 41, 41-53.

Roediger, H. L., and McDermott, K. B. (1996). False perceptions of false memories. J. Exp. Psychol. Learn. Mem. Cogn. 22, 814-816.

Rosas, J. M., and Bouton, M. E. (1997). Additivity of the effects of retention interval and context change on latent inhibition: toward resolution of the context forgetting paradox. J. Exp. Psychol. Anim. Behav. Process 23, 283-294.
Ruediger, S., Vittori, C., Bednarek, E., Genoud, C., Strata, P., Sacchetti, B. and Caroni, P. (2011). Learningrelated feedforward inhibitory connectivity growth required for memory precision. Nature $473,514-518$.

Samson, R., and Pare, D. (2005). Activity-dependent synaptic plasticity in the central nucleus of the amygdala. J. Neurosci. 25, 1847-1855.

Schafe, G., Nader, K., Blair, H., and LeDoux, J. (2001). Memory consolidation of Pavlovian fear conditioning: a cellular and molecular perspective. Trends Neurosci. 24, 540-546.

Schafe, G. E., Doyere, V., and LeDoux, J. E. (2005). Tracking the fear engram: the lateral amygdala is an essential locus of fear memory storage. J. Neurosci. 25, 10010-10014.

Shaban, H., Humeau, Y., Herry, C., Cassasus, G., Shigemoto, R., Ciocchi, S., Barbieri, S., van der Putten, H., Kaupmann, K., Bettler, B., and Luthi, A. (2006). Generalization of amygdala LTP and conditioned fear in the absence of presynaptic inhibition. Nat. Neurosci. 9, 1028-1035.

Siegel, S. (1975). Evidence from rats that morphine tolerance is a learned response. J. Comp. Physiol. Psychol. 89, 498-506.

Siegel, S. (1976). Morphine analgesic tolerance: its situation specificity supports a Pavlovian conditioning model. Science 193, 323-325.

Smith, S. M. (1979). Remembering in and out of context. J. Exp. Psychol. Hum. Learn. Mem. 5, 460.

Spear, N., and Riccio, D. C. (1994). Memory: Phenomena and Principles. Boston: Allyn and Bacon.

Spear, N.E. (1973). Retrieval of memory in animals. Psychol. Rev. 80, 163-194.

Squire, L. R. (1992). Memory and the hippocampus: a synthesis from findings with rats, monkeys, and humans. Psychol. Rev. 99, 195-231.

Sun, N., and Cassell, M. (1993). Intrinsic GABAergic neurons in the rat central extended amygdala. J. Comp. Neurol. $330,381-404$

Thomas, D. A., and Riccio, D. C. (1979). Forgetting of a CS attribute in a conditioned suppression paradigm. Animal Learn. Behav. 7, 191-195.

Thomas, D. R., and Burr, D. E. (1969). Stimulus generalization as a function of the delay between training and testing procedures: a reevaluation. J. Exp. Anal. Behav. 12, 105-109.

Thomas, D. R., and Lopez, L. J. (1962) The effects of delayed testing on generalization slope. J. Comp. Physiol. Psychol. 55, 541-544.

Thomas, D. R., Windell, B. T., Bakke, I., Kreye, J., Kimose, E., and Aposhyan, H. (1985). Long-term memory in pigeons: I. The role of discrimination problem difficulty assessed by reacquisition measures II. The role of stimulus modality assessed by generalization slope. Learn. Motiv. 16, 464-477.

Vigot, R., Barbieri, S., Bräuner-Osborne, H., Turecek, R., Shigemoto, R. Zhang, Y. P., Lujan, R., Jacobson, L. H., Biermann, B., and Fritschy, J. M. (2006). Differential compartmentalization and distinct functions of GABAB receptor variants. Neuron 50, 589-601.

Walker, D. L., Ressler, K., Lu, K-T., and Davis, M. (2002). Facilitation of conditioned fear extinction by systemic administration or intraamygdala infusions of D-cycloserine as assessed with fear-potentiated startle in rats. J. Neurosci. 22, 2343-2351.

Watson, J. B., and Rayner, R. (1920). Conditioned emotional reactions. J. Exp. Psychol. 3, 1-14.

Weinberger, N. M. (2007). Associative representational plasticity in the auditory cortex: a synthesis of two disciplines. Learn. Mem. 14 $1-16$.

Wilensky, A. E., Schafe, G. E., Kristensen, M. P., and LeDoux, J. E. (2006). Rethinking the fear circuit: the central nucleus of the amygdala is required for the acquisition, consolidation, and expression of Pavlovian fear conditioning. J. Neurosci. 26 , 12387-12396.

Wiltgen, B. J., and Silva, A. J. (2007). Memory for context becomes less specific with time. Learn. Mem. 14, 313-317.
Wiltgen, B. J., Zhou, M., Cai, Y., Balaji, J., Karlsson, M. G., Parivash, S. N., Li, W., and Silva, A. J. (2010). The hippocampus plays a selective role in the retrieval of detailed contextual memories. Curr. Biol. 20, 1336-1344.

Winocur, G., Frankland, P. W., Sekeres, M., Fogel, S., and Moscovitch, M. (2009). Changes in contextspecificity during memory reconsolidation: selective effects of hippocampal lesions. Learn. Mem. 16, 722-729.

Winocur, G., Moscovitch, M., and Sekeres, M. (2007). Memory consolidation or transformation: context manipulation and hippocampal representations of memory. Nat. Neurosci. 10, 555-557.

Wixted, J. T. (2004). The psychology and neuroscience of forgetting. Annu. Rev. Psychol. 55, 235-269.

Zhou, Y., and Riccio, D. (1994). Pretest cuing can alleviate the forgetting of contextual stimulus attributes. Learn. Motiv. 25, 233-244.

Zhou, Y., and Riccio, D. (1996). Manipulation of components of context: the context shift effect and forgetting of stimulus attributes. Learn. Motiv. 27, 400-407.

Conflict of Interest Statement: The authors declare that the research was conducted in the absence of any commercial or financial relationships that could be construed as a potential conflict of interest.

Received: 29 February 2012; accepted: 16 May 2012; published online: 01 June 2012.

Citation: Jasnow AM, Cullen $P K$ and Riccio DC (2012) Remembering another aspect of forgetting. Front. Psychology 3:175. doi: 10.3389/fpsyg.2012.00175

This article was submitted to Frontiers in Cognition, a specialty of Frontiers in Psychology.

Copyright (๑) 2012 Jasnow, Cullen and Riccio. This is an open-access article distributed under the terms of the Creative Commons Attribution Non Commercial License, which permits noncommercial use, distribution, and reproduction in other forums, provided the original authors and source are credited. 